\title{
Assessment of Relationship between Wilms' Tumor Gene (WT1) Expression in Peripheral Blood of Acute Leukemia Patients and Serum IL-12 and C3 Levels
}

\author{
Omran Rezai ${ }^{1,2}$, Ali Khodadadi ${ }^{1,2 *}$, Yuji Heike ${ }^{3}$, Ali Mostafai ${ }^{4}$, Nader Dashti \\ Gerdabi $^{1,2}$, Mohammad Rashno ${ }^{1,2}$, Zahra Abdoli ${ }^{1,2}$
}

\begin{abstract}
Background: Leukemia is a common cancer among children and adolescents. Wilms' tumor gene (WT1) is highly expressed in patients with acute leukemia. It is found as a tumor associated antigen (TAA) in various types of hematopoietic malignancies and can be employed as a useful marker for targeted immunotherapy and monitoring of minimal residual disease (MRD). In this regard, WT1 is a transcription factor that promotes gene activation or repression depending on cellular and promoter context. The purpose of this study was assessment of WT1 gene expression in patients with acute leukemia, measurement of IL-12 and C3 levels in serum and evaluation of the relationship between them. Materials and Methods: We evaluated the expression of WT1 mRNA using real-time quantitative RT-PCR and serum levels of IL-12 and C3 using ELISA and nephelometry in peripheral blood of 12 newly diagnosed patients with acute leukemia and 12 controls. Results: The results of our study showed that the average wT1 gene expression in patients was 7.7 times higher than in healthy controls $(\mathrm{P}<0.05)$. In addition, IL-12 $(\mathrm{P}=\mathbf{0 . 0 0 3})$ and $\mathrm{C} 3(\mathrm{P}<0.0001)$ were significantly decreased in the test group compared to controls. Conclusions: WT1 expression levels are significantly higher in patients compared with control subjects whereas serum levels of interleukin-12 and $\mathrm{C3}$ are significantly lower in patients. Wt1 expression levels in patients are inversely related with serum levels of IL-12 and C3.
\end{abstract}

Keywords: Acute leukemia - C3 - interleukin-12 - Wilms tumor gene expression

Asian Pac J Cancer Prev, 16 (16), 7303-7307

\section{Introduction}

Cancer is a major human health problem in the world. It is the second leading cause of death after cardiovascular diseases. Currently, one out of four death cases in the world is due to cancer. American Cancer Society registered approximately 312,559 death cases due to cancer in 2005 but this number has increased in recent years (Jemal et al., 2008). ALL and AML are most blood cancers in Iran (Zand et al., 2012). According to the latest report by Cancer Management Center of the State Department of Non-communicable Diseases, Iranian Ministry of Health and Medical Education in 2010, the incidence of leukemia among males and females in the country was 1330 and 2131 cases, Statistics from Khuzestan in the same year indicated 203 and 148 cases of death due to cancer in male and female patients, respectively (A, 2012).
Immunotherapy is a novel approach to treat cancer due to problems associated with the existing therapies. WT1 gene plays an important role in leukemic cells and is subject to increased expression in leukemia patients. In the present study, we attempted to apply a new approach in treatment of cancer using immunotherapy (Sugiyama, 2010; Nakajima et al., 2015).

WT1 gene encodes for 10 exons and generates a $3 \mathrm{~kb}$ mRNA. This mRNA is translated into a protein with 449 amino acids (Yang et al., 2007). The N-terminal domain of WT1 is comprised of proline-glutamine-rich sequences and is involved in RNA and protein interactions. The C-terminal domain of WT1 is composed of four Krupplelike Cysteine2Histidine2 zinc fingers involved in RNA and protein interactions, which permit binding to target DNA sequences (Lee and Haber, 2001; Scharnhorst et al., 2001). WT1 is expressed during mammalian embryonic 
development in many tissues, such as urogenital system, gonads, spleen, liver, brain, and spinal cord. However, its expression is restricted to a limited number of tissues, mainly the genitourinary system during adulthood (Pritchard-Jones et al., 1990; Caldon et al., 2008; Wagner et al., 2008).

WT1 is highly expressed in bone marrow or peripheral blood of a variety of leukemias in comparison to normal bone marrow and progenitor cells (Morrison et al., 2006; Iranparast et al., 2013). It is highly expressed in acute myeloid leukemia (AML) and acute lymphoid leukemia (ALL), myelodysplastic syndromes (MDS), chronic myeloid leukemia in blast crisis and leukemic cell lines (Rosenfeld et al., 2003). 80\% of lymphoid leukemias and $90 \%$ of myeloid leukemias, both adult- and childhood, have elevated WT1 expression levels (Miyagi et al., 1993).

Immune therapy using WT1 protein was initiated in 2001. WT1 peptide is an appropriate tumor marker for minimal residual disease in patients with leukemia. Presence of antibodies against wt1 and its CTL specific form have been spontaneously detected in patients with tumors. This may be due to high immunogenicity of this protein in leukemia (Mayo et al., 1999).

Various cytokines are increased or decreased in leukemia and other cancers in different stages (Carter et al., 2001; Ryningen et al., 2005). Therefore, their measurement is very important at different stages of disease and treatment steps. IL-12 is a on most potent cytokine with strong antitumor activity (Tugues et al., 2014), IL-12 increases the expression cytokines such as TNF- $\alpha$ IFN- $\gamma$, which leads to activation of CTL and NK cells against tumor cells (Abbas et al., 2007). This cytokine reducing in patients with AML. It inhibits the proliferation of tumor cells, causes induction of apoptosis and reduces angiogenesis (Xu et al., 2010).

C3 complement is a crucial protein in complement cascade and is responsible for functions of complement, froms a major host detection and clearnce of potential pathogens. Tumor cells in tumor tissue cause activation of complement, especially C3, which plays an important role in lysis of tumor cells (Jurianz et al., 1999; Okroj et al., 2008).

The aim of this study was to investigate the expression level of wt1 gene and measure the serum levels of IL-12 and $\mathrm{C} 3$ in patients with acute leukemia compared with healthy control subjects.

\section{Materials and Methods}

\section{Patients and materials}

A total of 12 PB samples were obtained from 12 newly diagnosed acute leukemia patients (8 AML and 4 ALL cases). Diagnosis of AML was based on cytomorphological findings and immunophenotypic characteristics of BM leukemic cells. The patients were at presentation stage and were not subject to any chemotherapeutic regimen at the time of sampling, which was the main criterion for inclusion in this study. The patients were selected from among those attending the Hematology and Oncology clinic of Emam Reza hospital affiliated to Kermanshah University of Medical Sciences. PB samples were collected from 12 normal healthy donors (with mean age of 28 years and age range 20-40) as control.

\section{RNA Extraction and cDNA Synthesis}

Total RNA was extracted from leukemic cells and normal PBMCs using RNA-PLUS reagent (Sanacolon) according to manufacturer's instructions. First strand cDNA was synthesized using $1 \mu \mathrm{l}$ of total RNA in $20 \mu \mathrm{l}$ reaction mixture consisting of 5x RT-PCR buffer, dNTPs, deionized sterile $\mathrm{H} 2 \mathrm{O}$ and $\mathrm{M}-\mathrm{Mulv}$ reverse transcriptase (Fermentase). Thereafter, the mixture was incubated at $42^{\circ} \mathrm{C}$ for 60 minutes followed by 5 minutes in $70^{\circ} \mathrm{C}$.

\section{Real Time PCR}

$20 \mathrm{ul}$ of reaction mixture was poured into each well consisting of 10ul of SYBR Green, 1ul of each of genespecific primers, $2 \mathrm{ul}$ of cDNA and $6 \mathrm{ml}$ RNase-free water. Real-Time PCR reaction in the real-time (Corbett) Rotorgene has a specific time and temperature program. The forward primer AGGGTACGAGAGCGATAACCACAC and reverse primer CTCAGATGCCGACCGTACAAGA were used for WT1. For GAPDH, the forward primer GCAAGAGCACAAGAGGAAGA and the reverse primer ACTGTGAGGAGGGGAGATTC were used.

\section{ELISA}

technique was used to prepare working solution for measurement of serum IL-12 )ebio science (according to manufacturer's instructions.

Nephelometry: Minineph human C3 kit )binding site) was used for measurement of $\mathrm{C} 3$ in serum of patients with acute leukemia and control subjects according to manufacturer's instructions.

\section{Statistical analysi}

Rest software was used for statistical analysis of data obtained from two Real Time PCR experiments. SPSS software (version 16: SPSS) and T-test were used for statistical analysis of serum IL-12 and C3 tests. The data revealed significant coefficients at $95 \%$ level.

\section{Results}

Real-time PCR experiment was performed after RNA extraction and synthesis of cDNA in healthy control subjects and patients. The results were analyzed using Rest software. Average WT1 gene expression in patients with acute leukemia was 7.7 times higher than healthy control subjects.

The results showed that WT1 gene expression was significantly increased in the test group $(\mathrm{P}<0.05)$ compared to control. (Figure 1)

ELISA was performed after separation of serum from healthy control subjects and acute leukemia patients. The results were analyzed by optimizer SPSS software. The T-test showed statistically significant results with $\mathrm{P}<0.05$. The results showed that IL-12 was significantly reduced in the test group $(\mathrm{P}=0.003)$ compared to control (Figure 2).

C3 level was measured in healthy control subjects and acute leukemia patients after separation of the serum. The results were analyzed using SPSS optimizer software. 


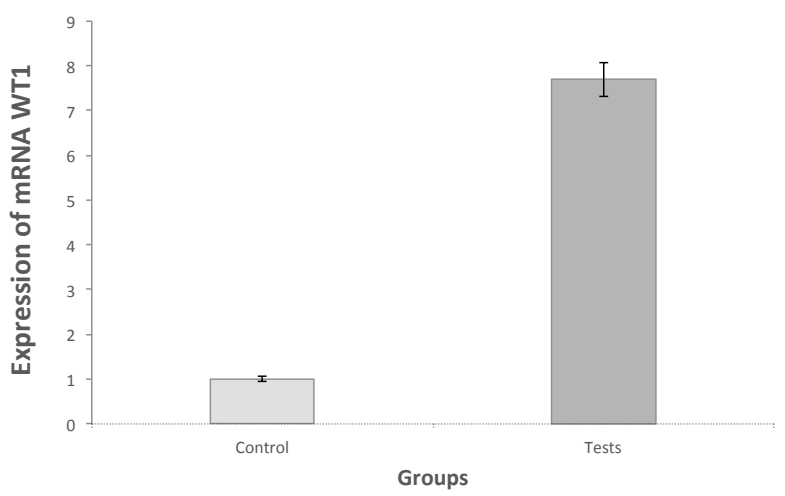

Figure 1. Fold Increase of mRNA WT1 Gene Expression Relative to Controls in Acute Leukemia. Significant difference between the controls and leukemia groups $(\mathrm{P}<0.05)$

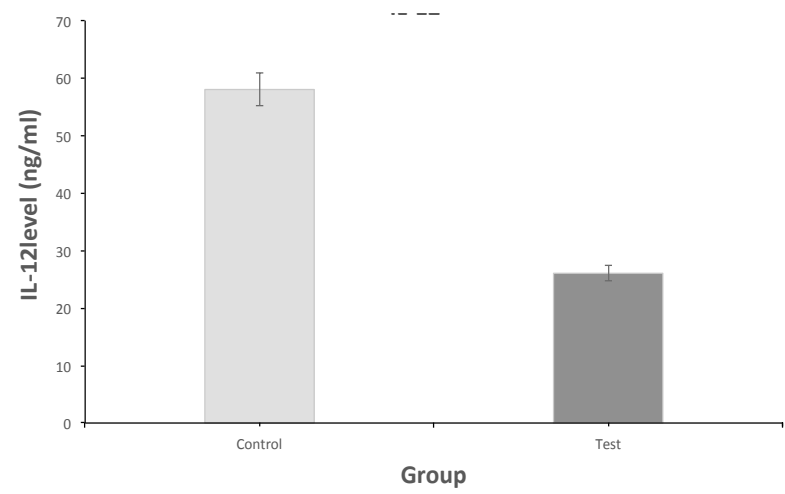

Figure 2. Serum Levels of IL-12 Ratio in Controls and

Acute Leukemia. Significant difference between the controls and acute leukemia groups $(\mathrm{P}=0.003)$

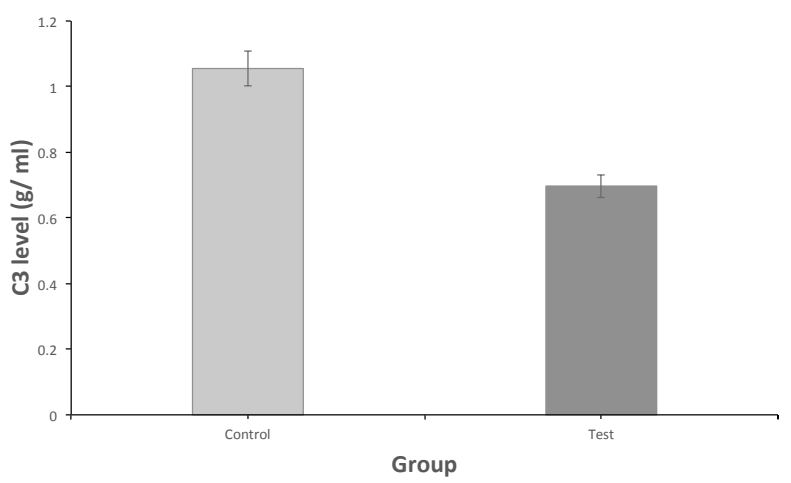

Figure 3. Serum Levels of C3 Ratio in Controls and Acute Leukemia. Significant difference between the controls and acute leukemia groups $(\mathrm{P}<0.0001)$

T-test showed statistically significant results with $\mathrm{P}<0.05$. Results showed that the level of C3 was significantly reduced in the test group $(\mathrm{P}<0.0001)$ compared to control (Figure 3).

\section{Discussion}

Recent advances in the field of molecular biology and tumor immunology have resulted in identification of a large number of tumor associated antigens (TAAs) and their immunogenic epitopes from various types of malignant neoplasms (Oka et al., 2004). Identification of TAAs may also be useful as targets for immunotherapy and molecular targeting therapy as well as development of diagnostic tools. WT1, currently known as a pan leukemic marker, is among TAAs expressed in various types of solid tumors and hematopoietic malignancies (Kletzel et al., 2002).

WT1 is highly expressed in bone marrow or peripheral blood of a variety of leukemias in comparison to normal bone marrow and progenitor cells (Miyagi et al., 1993). In general, the expression of WT1 varies between and within different forms of human leukemia (Yamamoto et al., 2007). In chronic myelogenous leukemia, WT1 levels are usually low in chronic phase but are frequently increased as patients progress towards accelerated and blast crisis phase of the disease (Menssen et al., 1995). In acute leukemias, increased level of WT1 can be found in both acute lymphoblastic and myeloblastic leukemia (Scharnhorst et al., 2001).

In our study, we determined the ratio of WT1 expression in PBMCs of normal subjects and patients with acute leukemia. Average WT1 gene expression in patients with acute leukemia was 7.7 times higher than healthy control subjects. Our findings are very similar to previous reports. Therefore, it can be concluded that WT1 gene expression is significantly different in patients compared to controls. Accordingly, WT1 gene expression in patients with acute leukemia can be used as an important peptide indicator for diagnosis and monitoring the course of treatment.

Combining the results of several studies, WT1 RNA levels, as assessed by RT-PCR and Northern blotting (earlier studies), were elevated in a total of 354 out of 476 (74\%) AML patient samples (Brieger et al., 1994; Schmid et al., 1997). In acute lymphoblastic leukemia, WT1 RNA was increased in 86 out of $131(66 \%)$ patient samples (Chiusa et al., 2006). High levels of WT1 were also observed in some forms of myelodysplastic syndromes (MDS). In MDS, increased WT1 expression is associated with higher blast counts and portends an early progression to AML (Tamaki et al., 1999).

Increased or decreased synthesis of various cytokines and their measurement in different stages of leukemia is important at different stages of disease and treatment. IL-12 is involved in the immune system with strong antitumor activity, which plays an important role in cancer $(\mathrm{Xu}$ et al., 2010) In our study, serum samples from patients with acute leukemia and healthy control subjects were subject to IL-12 measurement. The results showed that IL-12 was significantly reduced in the test group compared to control. Previous studies have showed the production of IL-12 decreased significantly in the patients with tumor metastases (Shibata et al., 2002; Pegram et al., 2014). Hera et al. reported that in the patients with advanced tumor stages had significantly decreased in IL-2 expression and serum level ofIL-12 (O'Hara et al., 1998). IL-12 can help increase the chance of disease prevention and treatment, inhibits tumor cell proliferation and decreases angiogenesis (Ferretti et al., 2010). According to a number of reports in several animal tumor models, IL-12 is not only able to prevent cancer but prevents the progression of tumor metastasis and may induce anti-tumor immunity (Opal and DePalo, 2000).

Complement activation plays a crucial role in 
Omran Rezai et al

defending the body against tumors. Measurement of the components of complement system, especially $\mathrm{C} 3$, is important in the diagnosis, evaluation and treatment of disease (Pio et al., 2014). The results of the present study show that C3 level was significantly reduced in leukemia patients compared to control. The complement protein C3 plays an important role in complement activity, including the destruction of tumor cells (Jurianz et al., 1999; Okroj et al., 2008). In a study conducted by Jurianz $\mathrm{K}$ et al in Germany in 1999, it was concluded that complement has anti-tumor effect (Jurianz et al., 1999).

In conclusion, The results of this research showed that Wt1 expression levels are significantly higher in patients with acute leukemia compared with healthy control subjects and Serum levels of IL-12 and C3 are significantly lower in patients than healthy control subjects. Wt1 expression levels in patients are inversely related with serum levels of IL-12 and C3 levels. Tumor-produced WT1 may regulate the IL-12 and C3 gene expression and decrease serum levels of IL-12 and C3 in patients with acute leukemia.

\section{Acknowledgements}

This work was supported by department of Immunology from Ahvaz Jundishapur University of Medical Sciences. All authors declare that they have no financial or nonfinancial conflicts of interest related to the subject matter or materials discussed in the manuscript.

\section{References}

A M (2012). Mesdaghinia. Reporting of cancer cases registered in 2008 and 2009 , Tehran - Iran. Cancer office, center for non-communicable diseases, department of health management 2012.(Presian)

Abbas AK, Andrew HL, Shiv P (2007). Cellular and molecular immunology. Sixth edition, SaundersElsevier, 580-618.

Brieger J, Weidmann E, Fenchel K, et al (1994). The expression of the Wilms' tumor gene in acute myelocytic leukemias as a possible marker for leukemic blast cells. Leukemia, 8, 2138-43.

Caldon C, Lee C, Sutherland R, et al (2008). Wilms' tumor protein 1: an early target of progestin regulation in T-47D breast cancer cells that modulates proliferation and differentiation. Oncogene, 27, 126-38.

Carter BZ, Milella M, Altieri DC, et al (2001). Cytokineregulated expression of survivinin myeloid leukemia. Blood, 97, 2784-90.

Chiusa L, di Celle PF, Campisi P, et al (2006). Prognostic value of quantitative analysis of WT1 gene transcripts in adult acute lymphoblastic leukemia. haematologica, 91, 270-1.

Ferretti E, Di Carlo E, Cocco C, et al (2010). Direct inhibition of human acute myeloid leukemia cell growth by IL-12. Immunology letters, 133, 99-105.

Iranparast S, Assarehzadegan M-A, Heike Y, et al (2014). Wilms' tumor gene (WT1) expression correlates with vascular epithelial growth factor (VEGF) in newly acute leukemia patients undergoing chemotherapy. Asian Pac J Cancer Prev, 15, 9217-23

Jemal A, Siegel R, Ward E, et al (2008). Cancer statistics, 2008. CA: A Cancer J Clin, 58, 71-96.

Jurianz K, Ziegler S, Garcia-Schüler H, et al (1999). Complement resistance of tumor cells: basal and induced mechanisms.
Molecular Immunol, 36, 929-39.

Kletzel M, Olzewski M, Huang W, et al (2002). Utility of WT1 as a reliable tool for the detection of minimal residual disease in children with leukemia. Pediatric and Developmental Pathology, 5, 269-75.

Lee SB, Haber DA (2001). Wilms tumor and the WT1 gene. Experimental Cell Res, 264, 74-99.

Mayo MW, Wang CY, Drouin SS, et al (1999). WT1 modulates apoptosis by transcriptionally upregulating the bcl-2 protooncogene. The EMBO J, 18, 3990-4003.

Menssen H, Renkl H-J, Rodeck U, et al (1995). Presense of Wilms' tumor gene (wt1) transcripts and the WT1 nuclear protein in the majority of human acute leukemias. Leukemia, 9, 1060-7.

Miyagi T, Ahuja H, Kubota T, et al (1993). Expression of the candidate Wilm's tumor gene, WT1, in human leukemia cells. Leukemia, 7, 970-7.

Morrison AA, Ladomery MR (2006). Presence of WT1 in nuclear messenger RNP particles in the human acute myeloid leukemia cell lines HL60 and K562. Cancer letters, 244, 136-41.

Nakajima H, Oka Y, Tsuboi A, et al (2015). Enhancement of efficacy of wilms'tumor gene WT1 Product-derived peptide cancer vaccine by co-administration with immunopotentiating agents: lessons from mouse models. in 'inflammation and immunity in cancer', Eds Springer, 165-83

O'Hara RJ, Greenman J, MacDonald AW, et al (1998). Advanced colorectal cancer is associated with impaired interleukin 12 and enhanced interleukin 10 production. Clin Cancer Res, 4, 1943-8.

Oka Y, Tsuboi A, Taguchi T, et al (2004). Induction of WT1 (Wilms' tumor gene)-specific cytotoxic T lymphocytes by WT1 peptide vaccine and the resultant cancer regression. Proceedings of the National Academy of Sciences of the United States of America, 101, 13885-90.

Okroj M, Hsu Y-F, Ajona D, et al (2008). Non-small cell lung cancer cells produce a functional set of complement factor I and its soluble cofactors. Molecular Immunol, 45, 169-79.

Opal SM, DePalo VA (2000). Anti-inflammatory cytokines. Chest Journal, 117, 1162-72.

Pegram H, Purdon T, van Leeuwen D, et al (2014). IL-12secreting CD19-targeted cord blood-derived T cells for the immunotherapy of B-cell acute lymphoblastic leukemia. Leukemia, 1-8.

Pio R, Corrales L, Lambris JD (2014). The role of complement in tumor growth. In 'Tumor Microenvironment and Cellular Stress', Eds Springer, 229-62

Pritchard-Jones K, Fleming S, Davidson D, et al (1990). The candidate Wilms' tumour gene is involved in genitourinary development. Nature, 346, 194-7.

Rosenfeld C, Cheever M, Gaiger A(2003). WT1 in acute leukemia, chronic myelogenous leukemia and myelodysplastic syndrome: therapeutic potential of WT1 targeted therapies. Leukemia, 17, 1301-12.

Ryningen A, Wergeland L, Glenjen N, et al (2005). In vitro crosstalk between fibroblasts and native human acute myelogenous leukemia (AML) blasts via local cytokine networks results in increased proliferation and decreased apoptosis of AML cells as well as increased levels of proangiogenic Interleukin 8. Leukemia research, 29, 185-96.

Scharnhorst V, van der Eb AJ, Jochemsen AG (2001). WT1 proteins: functions in growth and differentiation. Gene, 273, 141-61.

Schmid D, Heinze G, Linnerth B, et al (1997). Prognostic significance of WT1 gene expression at diagnosis in adult de novo acute myeloid leukemia. Leukemia, 11, 639-43.

Shibata M, Nezu T, Kanou H, et al (2002). Decreased production 

of interleukin-12 and type 2 immune responses are marked in cachectic patients with colorectal and gastric cancer. $J$ Clin Gastroenterol, 34, 416-20.

Sugiyama H (2010). WT1 (Wilms' tumor gene 1): biology and cancer immunotherapy. Japanese J Clin Oncol, 40,377-87.

Tamaki H, Ogawa H, Ohyashiki K, et al (1999). The Wilms' tumor gene WT1 is a good marker for diagnosis of disease progression of myelodysplnstic syndromes. Leukemia, 13, 393-9.

Tugues S, Burkhard S, Ohs I, et al (2014). New insights into IL-12-mediated tumor suppression. Cell Death \& Differentiation, 22, 237-46.

Wagner N, Panelos J, Massi D, et al (2008). The Wilms' tumor suppressor WT1 is associated with melanoma proliferation. Pflïgers Archiv-European J Physiol, 455, 839-47.

Xu M, Mizoguchi I, Morishima N, et al (2010). Regulation of antitumor immune responses by the IL-12 family cytokines, IL-12, IL-23, and IL-27. Clinical and Developmental immunology, 832454, 9.

Yamamoto S, Tsuda H, Kita T, et al (2007). Clinicopathological significance of WT1 expression in ovarian cancer: a possible accelerator of tumor progression in serous adenocarcinoma. Virchows Archiv, 451, 27-35.

Yang L, Han Y, Saiz FS, et al (2007). A tumor suppressor and oncogene: the WT1 story. Leukemia, 21, 868-76.

Zand AM, Imani S, Saadati M, et al (2012). Statistical approach to discovery of factors impacting on emergence of blood cancers in Iran. Asian Pac J Cancer Prev, 13, 5965-7. 